\title{
Fuzzy Adaptive Modified PSO-Algorithm Assisted to Design of Photonic Crystal Fiber Raman Amplifier
}

\author{
Majid Akhlaghi ${ }^{1 *}$ and Farzin Emami ${ }^{2}$ \\ ${ }^{1}$ Young Researchers and Elite Club, Omidieh Branch, Islamic Azad University, Omidieh, Iran \\ ${ }^{2}$ Department of Opto Electronic, Shiraz University of Technology, Shiraz, Iran
}

(Received January 24, 2013 : revised March 29, 2013 : accepted April 22, 2013)

\begin{abstract}
This paper presents an efficient evolutionary method to optimize the gain ripple of multi-pumps photonic crystal fiber Raman amplifier using the Fuzzy Adaptive Modified PSO (FAMPSO) algorithm. The original PSO has difficulties in premature convergence, performance and the diversity loss in optimization as well as appropriate tuning of its parameters. The feasibility and effectiveness of the proposed hybrid algorithm is demonstrated and results are compared with the PSO algorithm. It is shown that FAMPSO has a high quality solution, superior convergence characteristics and shorter computation time.
\end{abstract}

Keywords: Optical amplifier, Raman amplifier, Optimization algorithm

OCIS codes : (070.0070) Fourier optics and signal processing, (230.4480) Optical amplifiers

\section{INTRODUCTION}

Nowadays a Raman amplifier is considered as a key component in realizing the wide-band amplification due to the low-noise operation and optical amplification in any wavelengths [1]. Over the past few years, photonic crystal waveguides have been widely studied, modeled and fabricated due to their peculiar properties such as endlessly single-moded [2], high-nonlinearity [3, 4], overall controllable dispersion properties [5, 6], bandwidth improvement [7, 8] and enhanced coupling properties [9]. A new fiber called the holey fiber is a category of photonic crystal fibers (PCFs) and has been used in Raman amplifiers as the gain medium $[10,11]$. Such fibers consist of a pure silica core surrounded by a regular array of longitudinal air holes and can offer tight modal confinement. Thus they can provide an effective nonlinearity per unit length and can have the same order or higher than a conventional fiber. Their Raman efficiency defined as the Raman gain coefficient can be divided by the pump effective area and is expected to be much higher than that of a standard single-mode fiber (SMF). In [12], authors report the experimental study of the effect of a nonlinear PCF on the noise characteristics of a distributed Raman amplifier. There are two types of reported optimization work about the PCF RAs. In the first type, Raman gain is inherently flat or may possibly have optical amplification just by using one laser pump [13]; whereas in the second type, because of the gain non-uniformity, gain flatness can achieve by application of many pumps [14]. In triangular PCFs, gain can be increased by varying the geometrical parameters in the fiber cross section such as hole distances, pitch wavelength and their ratio and higher $\mathrm{GeO}_{2}$ percentages [15]. The present study aimed to propose a more powerful algorithm called fuzzy adaptive modified particle swarm optimization (FAMPSO) to choose pump power and pump wavelength for a $10 \mathrm{Km}$ long PC fiber with backward eight-pump configuration in order to create a flattened gain in the $\mathrm{C}$ and $\mathrm{L}$ bands. This method not only enhances the accuracy of design Raman amplifier parameters but also increases the reliability and speed of design parameters.

\section{METHODS}

Taking into account the loss, noise and Raman interaction, the optical power evaluation along the waveguide (z-direction) is determined by [15]:

\footnotetext{
*Corresponding author: m.akhlaghi@sutech.ac.ir

Color versions of one or more of the figures in this paper are available online.
} 


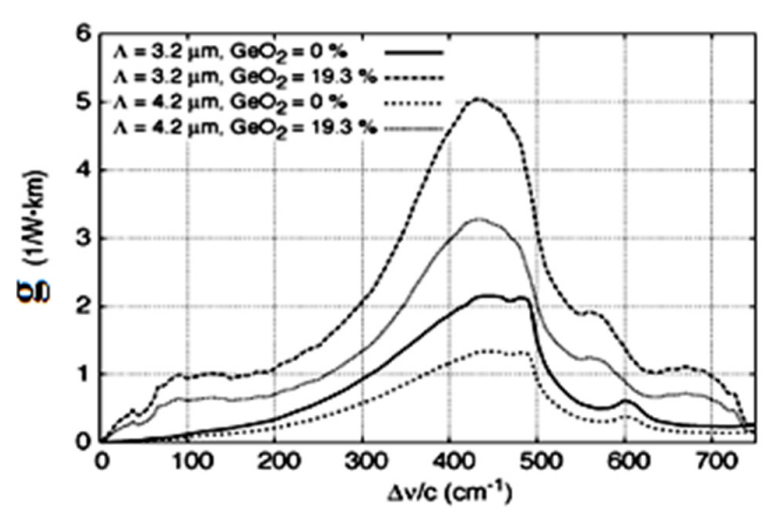

FIG. 1. Raman gain coefficient of PCF with different Pitch and Germanium mole fraction.

$$
\begin{aligned}
& \frac{d P_{v}^{ \pm}}{d z}=-\alpha_{v}^{ \pm}+P_{v}^{ \pm} \sum_{\mu>v} g_{R}(P+N)+P_{v}^{ \pm} \sum_{\mu<v} g_{R} 2 k_{p} E \\
& P=p^{+}+p^{-}, \quad N=n^{+}+n^{-}
\end{aligned}
$$

Where $p^{ \pm}\left(z, \lambda_{i}\right)$ are the forward or backward signal/pump powers at the wavelength, $\lambda_{i}, \alpha$ is the attenuated factor and $n^{ \pm}\left(z, \lambda_{i}\right)$ defines the forward or backward noise power which is due to the amplified Rayleigh backscattering and spontaneous Raman scattering effects. Raman gain $\gamma_{R}$ of this amplifier is shown in Fig. 1 [15].

\subsection{Classical PSO}

PSO is an optimization algorithm inspired by a habitation such as birds. This method is based on two points: artificial live and evolution. In the extension of PSO algorithm, the possible solutions of the desired optimization problem are regarded as non-bulky birds and any element of this colony called a particle. They can fly in an n-dimensional space and correct their trajectory based on the previous experiments as well as the neighboring particles. For particle number i, the displacement vector $\mathbf{X}_{\mathbf{i}}$ is defined as:

$$
\mathbf{X}_{\mathbf{i}}=\left[x_{i 1}, x_{i 2}, \ldots \ldots \ldots, x_{i n}\right]^{T} \in S
$$

Where $S$ is the searching space. This particle consists of the velocity vector $\mathbf{V}_{\mathbf{i}}$ in the form of:

$$
\mathbf{V}_{\mathbf{i}}=\left[v_{i 1}, v_{i 2}, \ldots \ldots \ldots, v_{i n}\right]^{T} \in S
$$

The best former position $\mathbf{P}_{\mathbf{i}}$ in the search space of $S$, is defined as:

$$
\mathbf{P}_{\mathbf{i}}=\left[p_{i 1}, p_{i 2}, \ldots \ldots \ldots, p_{i n}\right]^{T}
$$

Using these definitions, we can find the new positions by the aid of some weighting and learning factors which affect the algorithm searching procedure severely [16].

$$
\begin{aligned}
& V_{i}^{k+1}=w V_{i}^{k}+c_{1} r_{1}\left(P_{i}^{k}-X_{i}^{k}\right)+c_{2} r_{2}\left(P_{g}^{k}-X_{i}^{k}\right) \\
& X_{i}^{k+1}=X_{i}^{k}+V_{i}^{k+1}
\end{aligned}
$$

Where $V_{i}{ }^{k}$ is velocity of particle $\mathrm{i}$ at iteration $\mathrm{k}, w$ is inertia weight factor, $c_{1}, c_{2}$ are acceleration constants, $x_{i}{ }^{k}$ is the position of particle $\mathrm{i}$ at iteration $\mathrm{k}, P_{i}{ }^{k}$ is the best position of particle $\mathrm{i}$ until iteration $\mathrm{k}, P_{g}{ }^{k}$ is the best position of group until iteration $\mathrm{k}$ and finally $\mathrm{r} 1, \mathrm{r} 2$ are random numbers between 0 and 1 . There are three tuning parameters; $c_{1}, c_{2}$ and $w$ which can control the algorithm behavior strongly. We can use $w$ to affect the former velocity on the next velocity. Increasing $w$ causes an increment in searching ability and decreasing $w$ can continue an accurate searching in the previously scanned areas. So, a proper selection provides a compromise between the local and the global searches. In many works, the selected $w$ was big at first and after an initial search, this value would be lowered; a linearized model [17]. $c_{1}$ and $c_{2}$ are the best private and global positions, respectively. Since $c_{1}$ is the amount of particle trust in the previous experiments, it is called cognitive parameter, whereas $c_{2}$ describes the trust amount on the group and hence it is entitled the social parameter. For $c_{1}>c_{2}$ the particle is attracted to the best private position and the reverse is true for the other case. Many optimization procedures utilize $c_{1}=c_{2}=2$. To improve the PSO convergency, we reform and modify this algorithm by combining three algorithms; modified PSO, adaptive PSO and fuzzy PSO.

\subsection{Modified PSO (MPSO)}

One of the disadvantages of the original PSO is falling in a local optimum point, so in order to improve the convergence property and accuracy of the PSO algorithm, this paper presented a modified particle swarm optimization method. Premature convergence can take place under different situations such as the population has converged to local optima, the population has lost its diversity, and the search algorithm has proceeded slowly or hasn't proceeded at all. Mutation is a powerful strategy to increase the population diversity and improve the PSOs performance [18]. This paper employed a new mutation operator described as follows: In each iteration, three vectors are selected randomly $(Z 1, Z 2, Z 3)$ from the initial population after these vectors are mutated. It is worthwhile noting that for seeking the search space uniformly, the selected vectors should be different $(Z 1 \neq Z 2 \neq Z 3)$. A mutant vector $(X m u t)$ is generated as: [19]:

$$
\mathbf{X}_{m u t}=\mathbf{X}_{z 1}+\beta^{*}\left(\mathbf{X}_{z 2}-\mathbf{X}_{z 3}\right)
$$


Where $\beta^{*}$ is the mutation constant with the value of: $0.93<$ $\beta^{*}<1$. The target vector is mixed with the mutated vector, using the following scheme to yield the trial vector:

$$
\mathbf{X}_{n e w, i}=\left\{\begin{array}{cc}
\mathbf{X}_{m u t, i}, & \text { if }(\text { rand }<\text { crossover }) \\
\mathbf{X}_{i}, & \text { Otherwise }
\end{array} \quad i=1,2, \ldots \ldots ., n\right.
$$

Usual crossover is selected in the range of: $0.1<$ crossover $<0.9$. In this paper $\beta^{*}=0.93$ and crossover $=0.1$ [16].

\subsection{Adjusting the Leaming Coefficients}

Learning coefficients, $C_{1}$ and $C_{2}$, are used for determination of the local and overall best response in relation to particle rates, respectively. $C_{1}$ and $C_{2}$ in conventional PSO are considered as the constant value or change linearly during each iteration. The results show that these characteristics cannot obtain optimal results in some cases. So in this paper, these coefficients are computed as follows [17].

$$
c_{1,2}=1+\left[1+\exp \left(-\frac{G_{-} \text {Best_Value }}{G_{0}}\right)^{n}\right]^{-1}
$$

We chose $\mathrm{n}=2$ and $G_{0}$ as the $G_{-}$best_value in the first iteration.

\subsection{Fuzzy Formulation for Tuning of $w$}

Weight coefficient determines the effect of the past velocity experience on the present one. The right choice of this parameter results in the balance between global and local search of PSO algorithm. Great weight coefficient enhances the global search performance and small weight coefficient improves the local search. The parameter $w$ is often held constant or linearly changed for the entire run of a PSO but this method cannot obtain the global optima in many cases. The best choice for adjusting the computed weight coefficient is based on changes in G best value and in this regard the fuzzy logic method is applied. The inputs of the fuzzy method are NFV and $w$ while the output of this method is $\Delta w$ which are computed as follows [20]:

$$
N F V=\frac{\left(F V-F V_{\min }\right)}{\left(F V_{\max }-F V_{\min }\right)}
$$

In Eq. (11) FV is a current best performance evaluation and the calculated value of FV from Eq. 11 at the first iteration may be used as $F V_{\min }$ for the next iterations. $F V_{\max }$ is a very large value which is greater than any feasible solution. Usual inertia weight value is about $0 / 4<w<0 / 9$. In the fuzzy set, both positive and negative corrections are essential for the inertia weight. Therefore, for the inertia weight correction, a range of 0.1 to 0.1 has been selected [20].

$$
w^{k+1}=w^{k}+\Delta w
$$

For achieving the optimal point, objective functions should be described by membership functions. In this paper, for simplicity, all the membership functions are modeled with triangular shape and also they can take one of the three following status: Small (S), Medium (M) and Large (L). In Table 1, output variables of fuzzy sets are presented in three forms of linguistic values; NE (Negative), ZE (Zero), and PE (Positive) with related membership functions, as shown in Fig. 2. Rules of fuzzy sets are shown in Table 1. There are nine possible rules for two input variables and three linguistic values for all input variables. Each fuzzy rule as from an "IF-THEN" statement like the following rule, for example: If $N F V$ is $(L)$ and $w$ is $(M)$ then $\Delta w$ is $(Z E)$. The fuzzy rules are shown in Table 1 used to modify the inertia weight correction $(\Delta w)$. Each rule represents a relation between the input and output spaces. The membership functions are depicted in Fig. 2 are utilized for fuzzifying each input and output variable.

Based on the above theoretical description, we simulated

\begin{tabular}{|c|c|c|c|c|}
\hline \multirow{2}{*}{\multicolumn{2}{|c|}{$\Delta W$}} & \multicolumn{3}{|c|}{$W$} \\
\hline & & \multirow{2}{*}{$\begin{array}{c}S \\
Z E \\
\end{array}$} & \multirow{2}{*}{$\begin{array}{c}M \\
N E\end{array}$} & \multirow{2}{*}{$\begin{array}{c}L \\
N E\end{array}$} \\
\hline \multirow{3}{*}{$N F V$} & $S$ & & & \\
\hline & $M$ & $P E$ & $Z E$ & $N E$ \\
\hline & $L$ & $P E$ & $Z E$ & $N E$ \\
\hline
\end{tabular}
our optimization method by using FAMPSO as shown in the flowchart of Fig. 3.

TABLE 1. Fuzzy rules of the input and output variables
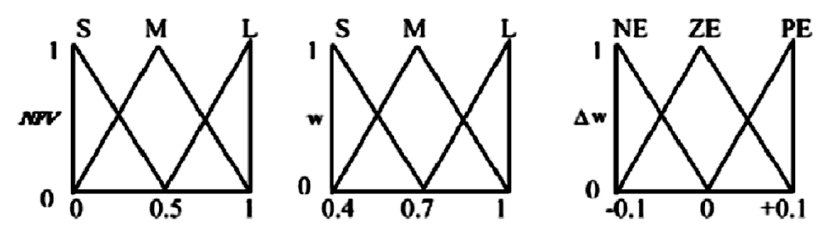

FIG. 2. The membership functions.

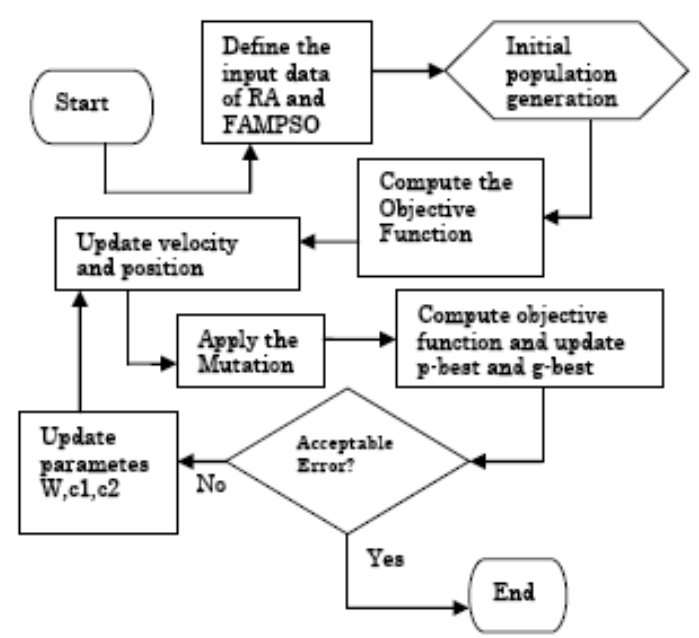

FIG. 3. Flowchart of FAMPSO algorithm. 


\section{NUMERICAL RESULTS}

Consider a WDM system with 16 -channels. There is a grid of $3.75 \mathrm{~nm}$ with signal powers of about $20 \mathrm{~mW}$. In this simulation we assumed that the lossy fiber has a length of about $10 \mathrm{Km}$. Runge-Kutta and a shooting method is used to solve the pump and signal interaction equations [21]. Our goal is to minimize the gain ripple in the C-L band by utilizing of the FAMPSO method to introduce optimizing sixteen parameters; the wavelengths and power levels for the 8 pumps range are from $1420 \mathrm{~nm}-1520 \mathrm{~nm}$ and $0 \mathrm{~mW}$ $120 \mathrm{~mW}$ respectively. For Optimizing gain ripple, the FAMPSO method should be minimizing the following function:

$$
F=\sum_{n=1}^{16}\left(G_{n}-\bar{G}\right)^{2}, \quad G_{n}=20 * \log \left(\frac{P(L)}{P(0)}\right)
$$

In this equation $\bar{G}$ is a predefined average gain for which minimization must be done around it. In this work, the particle numbers are three times of the optimization parameters. Initially any increases in the number of particles can improve the convergence speed, but accuracy and convergence speed will be reduced for more iterations. Application of the FAMPSO algorithm to optimize the Raman gain of a PCFRA is plotted in Fig. 4 for a system with eight backward pumps. As shown, variations of the gains restricted to a range of about $0.1 \mathrm{~dB}$, which is very much smaller than other reported methods such as the genetic algorithm [22] and PSO algorithm [23]. The optimization results for eight-pump PCFRA are shown in Table 2. As shown in this Table we can design a

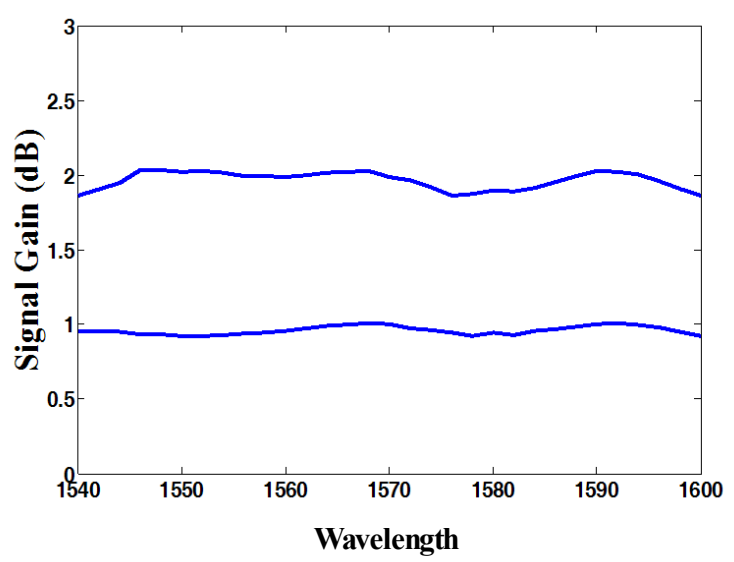

FIG. 4. Optimized Raman gain for eight backward pump using FAMPSO.

TABLE 2. Optimization results for eight-pumps of PCFRA

\begin{tabular}{|c|c|}
\hline \multirow{3}{*}{$\begin{array}{c}\text { FAMPSO } \\
\text { Gain around } \\
1(\mathrm{~dB})\end{array}$} & $\begin{array}{c}\mathrm{Pp}(\mathrm{mW}): 87.6 \quad 107.3 \quad 97.9 \quad 60.7 \quad 119.744 .657 .8 \\
102.3\end{array}$ \\
\hline & $\begin{array}{rlllll}\lambda \mathrm{p}(\mathrm{nm}): & 1469.3 & 1443.7 & 1469.9 & 1473.2 & 1507.7 \\
1511.6 & 1494 & 1489.6 & & \end{array}$ \\
\hline & Gain ripple $<0.1$ \\
\hline
\end{tabular}

uniform On-Off Raman gain with eight pumps for which the maximum powers is less than $120 \mathrm{~mW}$. Fig. 5 shows the gain ripples versus the number of iterations for the standard PSO and the proposed FAMPSO method. Indeed, the time consumption of the proposed method comparing with the classical PSO is shown in this figure. As shown in this figure, FAMPSO algorithm is performing better than PSO in terms of convergence speed and accuracy. Finally, Fig. 6

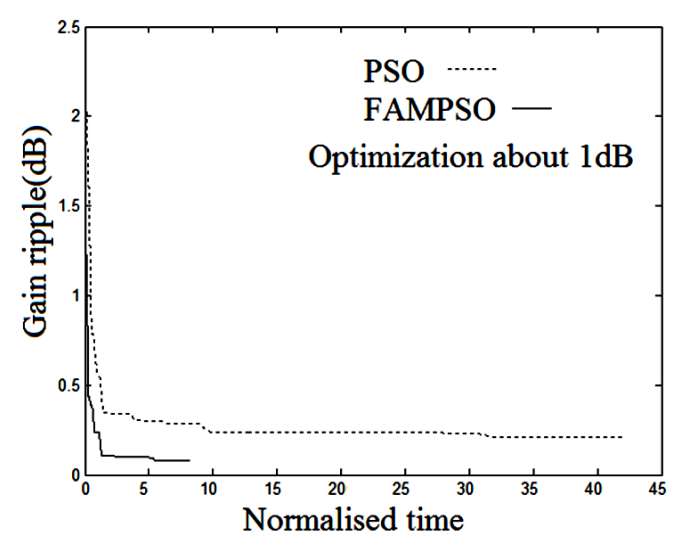

FIG. 5. Gain ripples versus the number of iteration for the standard PSO and the proposed FAMPSO.

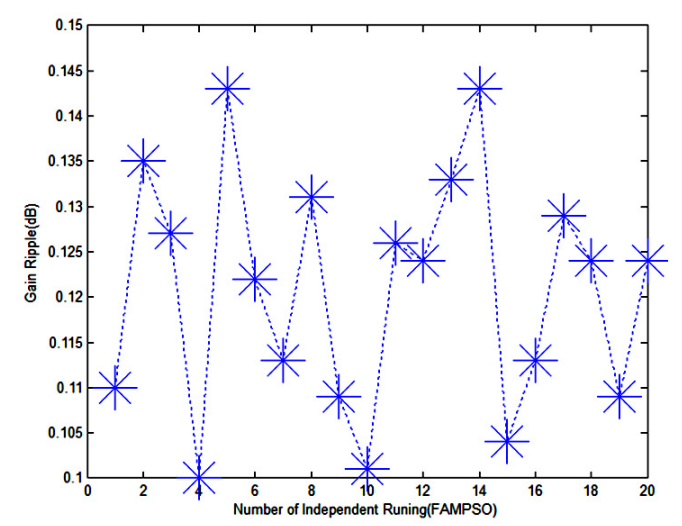

(a)

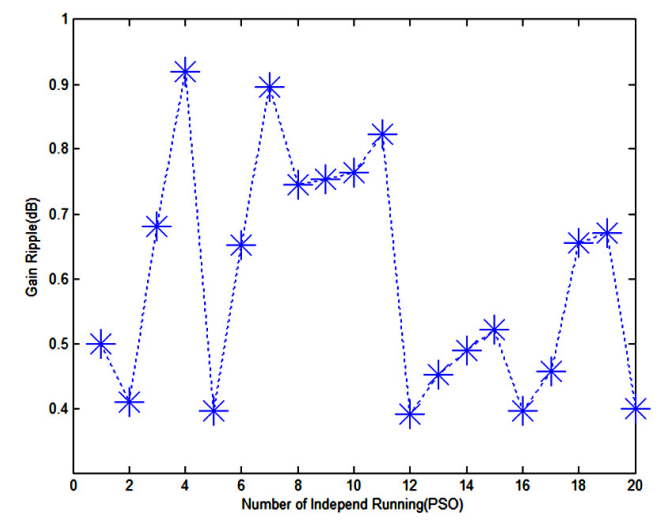

(b)

FIG. 6. Gain ripples versus the number of the independent algorithm running in (a) FAMPSO method, (b) PSO method. 
shows the development of the minimum gain ripples for 20 independent algorithms running which are obtained by PSO and FAMPSO algorithms. As it is seen, the FAMPSO method is more reliable than the classical PSO.

\section{CONCLUSION}

We simulated the optimum solution for a system of RA with eight backward pumps, for photonic crystal fiber we used a new optimization algorithm named FAMPSO. Designing a PCF-RA with a flattened gain done in the $\mathrm{C}$ and $\mathrm{L}$ band and the pump powers and the pump wavelengths were calculated so that a gain ripple of about $0.1 \mathrm{~dB}$ reached. This method not only enhances the accuracy of design Raman amplifier parameters but also increases the reliability and speed of design parameters.

\section{REFERENCES}

1. D. Wang, N. Zou, Z. Kang, J. Liu, Y. Namihira, and Y. Zhang, "Design and analysis of gain-flattened raman amplifiers with novel highly nonlinear photonic crystal fibers," in Proc. 7th Int. Conf. on WiCOM (shanghai, China, 2011), pp. $1-4$.

2. T. A. Birks, J. C. Knight, and P. S. J. Russell, "Endlessly single-mode photonic crystal fiber," Opt. Lett. 22, 961-963 (1997).

3. Z. Yusoff, J. H. Lee, W. Belardi, T. M. Monro, P. C. Teh, and D. J. Richardson, "Raman effects in a highly nonlinear holey fiber: amplification and modulation," Opt. Lett. 27, 424-426 (2002).

4. K. Saitoh and M. Koshiba, "Highly nonlinear dispersionflattened photonic crystal fibers for supercontinuum generation in a telecommunication window," Opt. Express 12, 2027-2032 (2004).

5. K. Saitoh, M. Koshiba, T. Hasegawa, and E. Sasaoka, "Chromatic dispersion control in photonic crystal fibers: application to ultra-flattened dispersion," Opt. Express 11, 843-852 (2003).

6. R. K. Sinha and S. K. Varshney, "Dispersion properties of photonic crystal fibers," Microw. Opt. Technol. Lett. 37, 129-132 (2003).

7. M. Danaie and H. Kaatuzian, "Bandwidth improvement for a photonic crystal optical Y-splitter," J. Opt. Soc. Korea 15, 283-288 (2011).

8. H. Aghababaeian, M. H. Vadjed-Samiei, and N. Granpayeh, "Temperature stabilization of group index in silicon slotted photonic crystal waveguides," J. Opt. Soc. Korea 15, 398-402 (2011).
9. M. Bozorgi and N. Granpayeh, "Directional emission from photonic crystal waveguide output by terminating with CROW and employing the PSO algorithm," J. Opt. Soc. Korea 15, 187-195 (2011).

10. C. J. S. de Matos, K. P. Hansen, and J. R. Taylor, "Experimental characterization of Raman gain efficiency of holey fiber," Electron. Lett. 5, 424-425 (2003).

11. C. L. Zhao, Z. Li, X. Yang, C. Lu, W. Jin, and M. S. Demokan, "Effect of a nonlinear photonic crystal fiber on the noise characterization of a distributed Raman amplifier," IEEE Photon. Technol. Lett. 3, 561-563 (2005).

12. S. K. Varshney, K. Saitoh, T. Fujisawa, and M. Koshiba, "Design of gain-flattened highly nonlinear photonic crystal fiber Raman amplifier using a single pump: a leakage loss approach," in Proc. OFC Conference (Anaheim, California, USA, 2006), paper OWD4.

13. J. Chang, T. Wang, and Z. Tao, "Optimal design of flattened gain spectrum of Raman amplifiers based on photonic crystal fibers," in Proc. Photonics and Optoelectronics, SOPO Symposium (Shanghai, China, 2009), pp. 1-4.

14. M. Bottacini, F. Poli, A. Cucinotta, and S. Selleri, "Modeling of photonic crystal fiber Raman amplifiers," J. Lightwave Technol. 7, 1707-1713 (2004).

15. F. Emami and M. Akhlaghi, "Gain ripple decrement of S-band Raman amplifier," IEEE Photon. Technol. Lett. 24, 1349-1352 (2012).

16. S. Chaoli, Z. Jianchao, and P. Jeng, "A modified particle swarm optimization with feasibility-based rules for mixedvariable optimization problems," Int. J. Innovative Computing, Information and Control 7, 3081-3096 (2011).

17. T. Niknam, H. Mojarrad, and M. Nayeripour, "A new fuzzy adaptive particle swarm optimization for non-convex economic dispatch," Int. J. Innovative Computing, Information and Control 7, 1764-1778 (2011).

18. T. Niknam, H. D. Mojarrad, and H. Z. Meymand, "A novel hybrid particle swarm optimization for economic dispatch with valve-point loading effects," Applied Energy Conversion and Management 52, 1800-1809 (2011).

19. P. Bajpai and S. N. Singh, "Fuzzy adaptive particle swarm optimization for biddinrg strategy in uniform price spot market," IEEE Trans. Power Sys. 22, 2152-2160 (2007).

20. Z. L. Dashtestani, F. Korushavi, and M. H. Rahmani, "An efficient shooting method for fiber amplifiers and lasers," J. Optics \& Laser Technology 40, 1041-1046 (2008).

21. G. C. M. Ferreira, S. P. N. Cani, M. J. Pontes, and M. E. V. Segatto, "Optimization of distributed Raman amplifiers using a hybrid genetic algorithm with geometric compensation technique," IEEE J. Photonics 3, 390-399 (2011).

22. H. M Jiang, K. Xie, and Y. F. Wang, "Shooting algorithm and particle swarm optimization based Raman fiber amplifiers," Opt. Commun. 283, 3348-3352 (2010). 